$\begin{array}{ll}\text { Italique } & \text { Italique } \\ \text { Poésie italienne de la Renaissance }\end{array}$

IX $\mid 2006$

Varia

\title{
Les Asolani de Pietro Bembo, ou le double portrait de l'amour
}

Lina Bolzoni

\section{(2) OpenEdition}

Journals

\section{Édition électronique}

URL : http://journals.openedition.org/italique/103

DOI : $10.4000 /$ italique. 103

ISSN : 1663-4438

Éditeur

Librairie Droz

Édition imprimée

Date de publication : 1 juin 2006

Pagination : 9-27

ISBN : 2-600-01088-2

ISSN : 1423-3983

Référence électronique

Lina Bolzoni, «Les Asolani de Pietro Bembo, ou le double portrait de l'amour », Italique [En ligne], IX | 2006, mis en ligne le 31 décembre 2009, consulté le 01 mai 2019. URL : http://

journals.openedition.org/italique/103; DOI : 10.4000/italique.103

(C) Tous droits réservés 
Lina BOLZONI

LESASOLANI DE PIETRO B E B O,

$\mathrm{O} \mathrm{U}$

LE D O UBLE PORTRA I T DE L'A M O U R 



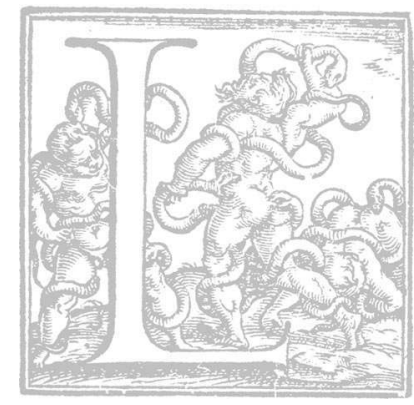

Es Asolani sont publiés en Isos à Venise

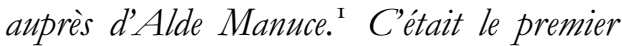
livre en langue vulgaire publié par Bembo, un jeune patricien vénitien encore à la recherche de sa voie, hésitant encore entre le latin et la langue vulgaire, entre la carrière politique et l'otium littéraire. ${ }^{2}$ Ce n'était pas le premier résultat de sa collaboration avec Alde Manuce, le grand éditeur qui avait commencé son activité à Venise en I494. Bembo avait déjà publié avec lui un dialogue latin sur l'Etna en I495 et, surtout, il avait inauguré le nouveau siècle avec les éditions du Canzoniere de Pétrarque (ISOI) et de la Commedia de Dante (I502): deux éditions novatrices du fait des critères philologiques adoptés et de la présentation; deux éditions qui influenceront longtemps l'écriture littéraire. Les Asolani tirent leur titre, selon le modèle cicéronien, de Asolo, localité où a lieu le dialogue que le texte relate; le thème central en est l'amour, sa nature multiple, l'attitude correcte que l'bomme doit avoir à son égard. L'ouvrage naît d'une longue gestation: commencé dans les dernières années $d u X V^{e}$ siècle, il a été publié, comme nous le disions, en I505; une nouvelle version du texte, revu et corrigé selon les canons linguistiques énoncés dans les Prose della volgar lingua, sort en I530. Cette position chronologique du texte - à cheval sur deux siècles - a une valeur emblématique.

Les Asolani sont le reflet, en effet, des projets et des expériences très différentes de la vie de Bembo: leur début s'inspire de la fin malheureuse d'un amour; de I500 à I502, l'écriture de l'ouvrage est liée de très près à l'amour que l'auteur éprouve pour Maria Savorgnan; les lettres que les deux amants échangent montrent que les parties les plus sensuellement inquiétantes de l'ouvrage - telle la description du plaisir que l'amant tire des larmes de sa dame - faisaient partie du jeu érotique des deux personnages. ${ }^{3}$ En I503 commence l'bistoire d'amour avec Lucrèce Borgia, que Bembo connaît à la Cour de Ferrare en qualité d'épouse d'Alphonse d'Este. ${ }^{4}$ Lucrèce devient la nouvelle destinataire des raisonnements d'amour des Asolani; c'est à elle qu'ils sont dédiés quand ils viennent à la lumière en ISOS ou plutôt c'est à elle que sont dédiés quelques-uns des exemplaires qui sont parvenus jusqu'à nous. ${ }^{5}$ La première édition de l'ouvrage a donc un double statut, caractérisé par la présence/absence de la lettre de dédicace; de même, le ton et le contenu de la lettre font naître, comme nous le verrons, un jeu de perspective, un effet déroutant de dédoublement.

Les Asolani réalisent en outre une synthèse complexe de traditions et de modèles différents, littéraires et philosophiques, anciens et modernes, latins et en langue vulgaire. Ainsi la tradition du dialogue latin, classique et buma- 
niste, est réécrite en vulgaire et utilisée également comme cadre et comme commentaire à un choix de poésies; si le cadre narratif renvoie à son tour au Décaméron, le choix de poésies s'inspire de plus en plus du Pétrarque du Canzoniere. Différentes traditions philosophiques - en particulier la réflexion moderne sur l'amour des néoplatoniciens florentins - sont utilisées pour donner une nouvelle dignité théorique à la tradition lyrique en vulgaire et en même temps pour réaliser une difficile réconciliation entre vie et littérature, entre autobiographie et création d'un modèle idéal.

Ces dialogues qui parlent d'amour en langue vulgaire, tout en proposant en même temps un modèle institutionnel de langue littéraire et de savoir-vivre social, s'imposeront comme un texte exemplaire. L'édition de I530, à côté de celle $d u$ Cortegiano, en I528, et de l'édition définitive du Roland Furieux, en I532, représente en effet une ligne de partage dans la riche production d'ouvrages consacrés à l'amour et aux femmes au XVI siècle. ${ }^{6}$

La célébrité des Asolani n'est pas seulement italienne: une traduction française, par Jean Martin, est publiée à Paris en I545 et elle sera réimprimée plusieurs fois au cours du XVI siècle. En outre, il ne faut pas oublier que Bembo est le protagoniste du dernier livre du Cortegiano grâce justement à l'autorité dont il jouissait pour avoir écrit les Asolani; la célébrité européenne $d u$ Cortegiano contribue à amplifier aussi la renommée de notre texte.

Mais revenons à sa première publication.

Le texte des Asolani, confié aux éditions prestigieuses d'Alde Manuce, offre une structure qui cache le travail complexe de gestation; la perfection de la géométrie exorcise, en d'autres termes, la diversité des modèles, le fouillis d'expériences que le texte contient. Les attentes du lecteur sont organisées autour d'un nombre magique, détenteur depuis toujours de la perfection: le nombre 3. Dans trois livres et en trois journées, trois jeunes lettrés parleront d'amour en présence de trois jeunes femmes; la localité est Asolo, comme nous l'avons déjà rappelé, en particulier la splendide demeure où Caterina Corner, reine de Chypre, célèbre le mariage d'une de ses demoiselles de Cour. Le lecteur est guidé dans un parcours exemplaire: chacun des trois livres ne sera que le développement d'un élément qui a été suggéré, esquissé initialement: trois demoiselles au service de la reine chantent chacune une chanson. Les deux premières chansons illustrent respectivement les souffrances et les joies procurées par l'amour, alors que la troisième propose une conception différente de l'amour, laquelle pourrait rétablir l'âge d'or.

\section{I}

Io vissi pargoletta in festa e 'n gioco, de' miei pensier, di mia sorte contenta: hor sì m'afflige Amor et mi tormenta, c'homai da tormentar gli avanza poco. 
Credetti, lassa, haver gioiosa vita

Da prima entrando, Amor, a la tua corte;

et già n'aspetto dolorosa morte:

o mia credenza, come mi hai fallita.

Mentre ad Amor non si commise anchora, vide Colcho Medea lieta e secura; poi ch'arse per Iason, acerba et dura fu sua vita infin a l'ultim'hora.

Enfant, je vécus au milieu des fêtes et des jeux, heureuse de mes pensées et contente de mon sort; à présent Amour m'afflige et me tourmente à tel point qu'il me tourmentera désormais pour peu de temps. Malheureuse que je suis, je crus avoir une vie pleine de joie lorsque j'entrai, Amour, pour faire partie de ta cour; à présent j'attends une mort douloureuse: oh combien j'ai été trompée par ce en quoi je croyais! Tant qu'elle ne s'était pas confiée à l'Amour, Médée vivait heureuse et en sûreté en Colchide; une fois qu'elle brûla pour Jason, sa vie devint amère et difficile jusqu'à son dernier soupir.

II

Io vissi pargoletta in doglia e 'n pianto, de le mie scorte et di me stessa in ira; hor sì dolci pensieri Amor mi spira, ch'altro meco non è che riso et canto.

Harei giurato, Amor, ch'a te gir dietro fosse proprio un andar con nave a scoglio; così là 'nd'io temea danno e cordoglio, utile scampo a le mie pene impetro. ${ }^{8}$

Infin quel dì, che pria la punse Amore, Andromeda hebbe sempre affanno et noia; poi ch'a Perseo si diè, diletto et gioia seguilla viva, et morta eterno honore.

Enfant je vécus dans la douleur et les pleurs, odieuse à ceux qui me tenaient compagnie et à moi-même; à présent Amour m'inspire des pensées si douces qu'autour de moi il n'y a que rires et chansons. J'aurais juré, Amour, que te suivre aurait été comme aller sur un écueil avec un navire: ainsi là où je craignais la souffrance et la douleur, 
j'ai trouvé la façon d'échapper à mes peines. Andromède vécut dans l'angoisse et l'ennui tant qu'Amour ne la blessa pas; après s'être donnée à Persée, vivante, elle obtint plaisirs et joies, et morte, honneur éternel.

III

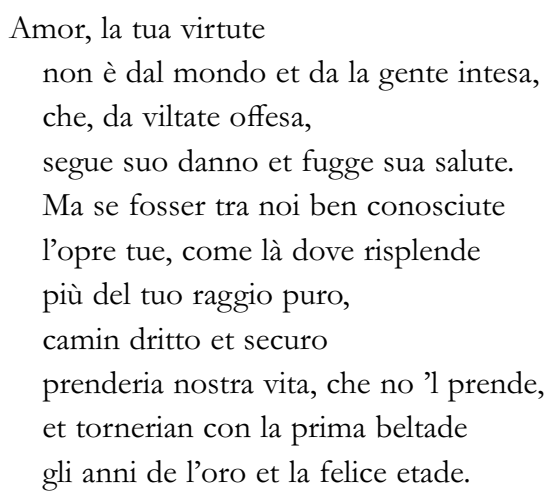

Voyons les deux premières chansons. Il s'agit de trois strophes qui, comme l'écrit Dionisotti, «n'ont rien métriquement de la chanson, et qui ne sont pas vraiment des madrigaux», c'est pourquoi on peut parler de deux chansonsodes. ${ }^{9}$ Elles sont liées par un rapport spéculaire: il s'agit dans les deux cas d'un récit exemplaire où l'expérience amoureuse renverse les attentes. Les deux premiers quatrains, divisés de manière régulière en deux distiques, marquent le contraste entre le passé et le présent, entre ce que l'on croyait et ce que l'on est en train de vivre. Le dernier quatrain garde cette structure et fait correspondre l'histoire que le «je» raconte à celle d'un personnage mythologique: au sort malbeureux de Médée dans le premier cas, à la joie et à la renommée qui revinrent à Andromède dans le second.

Le schéma métrique change avec le troisième texte (il s'agit d'une strophe de chanson utilisée comme un madrigal): les propos ne sont plus tenus à la première personne et une citation dantesque évidente ("da viltate offesa») Enfer II, 45) souligne le caractère moralement plus élevé de la poésie. L'opposition se situe à présent entre qui connaît la nature véritable d'Amour et qui, ne la connaissant pas, se condamne au malheur et à l'erreur. Les vers de la fin, qui chantent un retour possible à l'áge d'or, projettent les choix individuels sur un plan universel, qui implique toute l'bumanité et peut-être même le cycle cosmique.

Les trois demoiselles chantent accompagnées d'un instrument (les deux premières, d'un luth, la troisième, d'une viole). Il existe une correspondance de 
structure accentuée dans les deux premières chansons: la 'diversite' de la dernière chanson est préparée par la différence d'instrument ainsi que par d'autres signes de supériorité esthétique et morale manifeste, qui concernent aussi bien l'émetteur du message que les effets suscités chez le destinataire: la demoiselle est «bellissima sopra modo et per giudicio d'ogniun che la vide più assai che altra che in quelle nozze v'havesse» ("d'une beauté extraordinaire et selon le jugement de tous ceux qui la virent, bien plus belle que toutes celles qui étaient présentes au mariage"); son chant est tel que «alla dolce fiamma che le sue note ne' cuori degli ascoltanti lasciarono, quelle delle due fanciulle furono spenti e freddi carboni》 (I, III, D. 2I7: "ses notes enflammèrent agréablement le cour de ceux qui l'écoutèrent alors que celles des deux jeunes filles ne furent que cendres éteintes et froides"). Enfin, elle a un rapport privilégié avec la reine: c'est la reine elle-même qui lui a ordonné de chanter.

Le chant des trois jeunes filles est, à la fois, prélude et synthèse de l'ouvrage: si Perottino parle contre l'amour dans le premier livre, dans le deuxième Gismondo en chantera les louanges, alors que, dans le troisième livre, Lavinello invite à distinguer entre les différentes sortes d'amour, à se tenir dans les limites de la morale et indique à la fin une autre conception de l'amour où les composantes néoplatoniciennes sont très fortes. Le texte luimême s'empresse de signaler au lecteur la fonction structurale des trois chansons du début. En effet, quand Lavinello commence à parler, il s'adresse à la reine et déclare:

Questo poco, Madonna, che io v'ho fin qui detto, sarebbe alle nostre donne potuto per aventura bastare per dimostramento della menzogna che l'uno et l'altro de' miei compagni sotto le molte falde delle loro dispute haveano questi giorni, sì come udito havete, assai acconciamente nascosa; ma non a voi, né pure alla vostra fanciulla, che così vagamente l'altr'hieri alle tavole di Vostra Maestà cantando, ci mostrò quello che io dire ne dovea, poscia che i miei compagni, per le pedate dell'altre due mettendosi, haveano a tacerlo.

(III, XI, p. 329: "Ma Dame, les quelques mots que je vous ai dits jusque là auraient peut-être pu suffire à nos dames pour démontrer les mensonges que l'un et l'autre de mes compagnons avaient très habilement cachés sous les plis et les replis de leurs discussions, mais ils ne pouvaient les cacher ni à vous ni à votre demoiselle; celle-ci nous montra, en chantant de façon admirable avant-hier à la table de Votre Majesté, ce que je devais en dire, parce que mes deux compagnons qui suivaient les traces des deux autres demoiselles ne l'avaient pas dit").

La scène initiale doit donc rester présente dans la mémoire du lecteur: elle est l'image exemplaire qui se développera dans la suite du texte; elle est le caur de l'inventio et le reste de l'ouvrage en est, par certains aspects, l'exégèse. 
Le troisième livre se présente comme le moment de la vérité dévoilée. Selon les règles du code rbétorique, à un contenu plus élevé correspondront à la fois un public plus élevé - la reine sera présente aux «ragionamenti» ("raisonnements") - et un interlocuteur investi d'une autorité particulière: Lavinello se fait le porteur d'un message qu'un ermite lui a confié, un romito, un "ermite" qui vit au baut d'une colline. Ainsi, même les différents lieux se recomposent en une unité supérieure. Pour parler d'amour, les jeunes gens se sont séparés des autres, ils se sont installés dans cet endroit interne/externe à la Cour qu'est le jardin; ${ }^{\circ}$ la présence de la reine rétablit, de façon visuelle aussi, le contrôle sur un espace qui, ne fût-ce que de façon temporaire, s'était constitué comme séparé. Lavinello recompose également les contrastes et les limites de la discussion précédente sur l'amour, rapportant ainsi à l'intérieur de l'espace de la Cour un 'autre' point de vue, supérieur mais proche: l'ermite se trouve au haut d'un coteau, dit Lavinello, "che c'è qui dietro» ("qui est là derrière") et une vision l'a fait participer idéalement à ce qui est arrivé dans le jardin.

La structure ternaire des Asolani montre une perfection géométrique, elle crée une image où tout se tient; en même temps, avec ses jeux compliqués de perspective, elle semble nous inviter à chercher autre chose, elle nous pousse à entrer dans les coulisses, là où les règles et les trucages de la mise en scène se manifestent de façon plus évidente. En effet, le texte construit une machine optique complexe, il nous invite à ne pas nous contenter de la vision ordonnée, à deux dimensions, que l'ordre de la lecture et la succession des pages nous suggèrent. Essayons de déceler tout d'abord quel est le point de vue multiple que le texte construit sur lui-même, puis quelles suggestions figuratives interviennent dans cette opération.

Avant tout, nous devons remarquer que la structure que nous avons décriteune structure double, où les trois scènes initiales, c'est-à-dire les trois jeunes filles qui chantent, résument de façon emblématique les trois livres qui suivront - est le fruit d'un changement de mise en scène. Celle-ci apparait, en effet, dans la version imprimée, alors que dans la version manuscrite précédente, Gismondo invitait ses amis dans le jardin; il évoquait les deux chansons qui louaient et critiquaient l'amour et que deux jeunes filles avaient chantées en présence de la reine; chansons dont il tirait le sujet qu'il proposait au groupe. ${ }^{\mathrm{I}}$ Il s'agit donc d'une structure à laquelle l'auteur attribue une importance particulière. Dans sa version définitive, cette structure prend un rythme ternaire, elle renonce au flash back, c'est-à-dire à l'ordo artificialis du récit et le remplace par une scansion temporelle en deux temps qui se reflètent l'un dans l'autre: en effet, les trois chansons des jeunes filles et les propos des trois jeunes hommes se correspondent, ils renvoient les uns aux autres; de plus, les scènes des dialogues, bien plus amples et plus analytiques, se placent, de façon idéale, derrière les trois scènes du début et en constituent la toile de fond. Nous 
nous limiterons à mentionner que cette structure est l'un des signaux qui incitent à lire de façon différente le contenu réel de l'ouvrage. Plus qu'inviter à l'amour néoplatonicien, les Asolani mettent en scène la nature même de la littérature, sa capacité de donner forme à ce qui peut être dit, de jouer avec ses différentes possibilités expressives. Les poìtes expérimentent les différentes formes des images, affirme par exemple Gismondo; s'ils parlent de l'amour en termes de douleur même lorsque leur amour est partagé, ils le font «per porgere diversi suggeti agl'inchiostri, acciò che con questi colori $i$ loro fingimenti variando, l'amorosa pintura riesca agli occhi de' riguardanti più vaga» (II, VIII, p. 270: "pour offrir différents thèmes à l'écriture, afin que, variant leurs inventions avec ces couleurs, l'amoureuse peinture apparaisse plus belle aux yeux de ceux qui regardent').

"L'amorosa pintura»: cette expression, insérée dans un contexte s'inspirant fortement de la perception visuelle, remonte au canon de l'ut pictura poesis, qui est d'ailleurs largement présent dans le texte de Bembo. Ainsi au début du livre III, la reine demande à Bérénice de lui faire un résumé des propos tenus les jours précédents et s'apprête à écouter en personne la suite: «La Reina, uditola et parendole la macchia et l'ombra haver veduta di belle et convenevoli dipinture, sentendo che Lavinello havea a dire il dì seguente, si dispose di volerlo udire anchora essas) (III, II, p. 3Is: "La Reine l'écouta et comme il lui sembla avoir vu l'esquisse et l'ombre de très belles peintures, sachant en outre que Lavinello parlerait le lendemain, elle décida d'aller l'écouter elle aussi'). Nous pouvons alors accepter, pour ainsi dire, la provocation visuelle et nous demander si, au-delà du jeu de la variation des images que l'écriture crée à travers les métaphores, il n'y a pas quelque chose en plus, en d'autres termes, s'il n'y a pas aussi des suggestions figuratives qui concernent la structure même des Asolani. ${ }^{12}$ Comme nous l'avons déjà souligné, cette structure est élaborée, elle prend forme après des changements radicaux entre les dernières années $d u$ $X V^{e}$ siècle et les premières années du XVI $T^{e}$ siècle, et reste inchangée jusque dans les années I530. Dans la version définitive, nous avons une perspective complexe qui nous pousse à placer, de façon idéale, les trois dialogues derrière les trois scènes du début. Nous pourrions dire, en utilisant une terminologie empruntée aux arts figuratifs, que Bembo construit un triptyque dont chacune des scènes (les trois dialogues) est cachée par un panneau contenant à son tour une image qui renvoie de façon allusive à la scène à laquelle elle correspond: quelque chose de semblable, donc, aux portraits avec un pannean qui les recouvre ou avec un revers, des portraits produits entre le $X V^{e}$ et le $X V I^{e}$ siècle. ${ }^{13}$

Je me limiterai à rappeler ici quelques exemples parmi les plus célèbres. Tout d'abord les portraits des Ducs d'Urbin de Piero della Francesca, qui se trouvent à la Galerie des Offices à Florence. ${ }^{\mathrm{I}}{ }^{4}$ Federico da Montefeltro et son 
épouse Battista Sforza sont représentés de profil, de telle sorte que leurs figures dominent nettement le paysage, transmettant ainsi au spectateur une forte impression de majesté, de distance inaccessible. À chacun des deux portraits correspond un volet qui porte une scène de triomphe. Federico est assis sur un char, couronné par la Renommée. Et on peut lire cette inscription élégante en lettres capitales:

Clarus insigni vehitur triumpho quem parem summis ducibus perhennis fama virtutum celebrat decenter sceptra tenentem ("Glorieux celui que la renommée éternelle des vertus célèbre comme étant l'égal des grands condottieri; il est porté noblement en triomphe et il est digne de porter le sceptre”).

Son épouse Battista est assise sur le char triomphal de la Modestie et on peut lire cette inscription:

Que modum rebus tenuit secundis coniugis magni decorata rerum laude gestarum volitat per ora cuncta virorum ("Celle qui, les circostances étant favorables, sut rester mesurée est ornée des louanges engendrées par les entreprises que son noble mari a réalisées, elle est à présent louée par tous les hommes”).

Le dyptique de Piero della Francesca construit un spectacle en deux temps: celui qui regarde/lit doit accomplir un parcours qui lui permet de voir aussi bien le portrait des deux personnages, que le fait qu'ils incarnent les modèles exemplaires de la Renommée et de la Modestie.

Le deuxième exemple est celui du portrait de Ginevra de' Benci, attribué à Leonardo da Vinci, un portrait que l'on situe aujourd'bui entre 1478 et I480 et qui est conservé à la National Gallery de Washington. ${ }^{15}$ Cette fois, l'autre image est peinte au dos: une branche de genevrier est couronnée par une branche de laurier et une de palmier. Un cartouche unit entre elles les trois branches et porte l'inscription: "Virtutem forma decorat" ("La beauté est l'ornement de la vertu'). Il s'agit d'un emblème qui veut exalter les qualités physiques et morales de la jeune fille représentée. Le genevrier qui apparaît aussi dans le portrait proprement dit fait allusion au nom de la jeune fille, Ginevra. Il s'agit donc d'une interpretatio nominis, un expédient typique de la poésie élogieuse et amoureuse. Dans ce cas, le parcours que le spectateur doit accomplir lui permettra de voir, outre le portrait physique de Ginevra, le portrait moral qui est confié à l'emblème, au jeu entre l'image et le mot que l'emblème crée.

Le troisième exemple est le portrait de l'évêque Bernardo de' Rossi de Lorenzo Lotto. ${ }^{16}$ Le volet nous propose une image difficile à interpréter, qui semble représenter, à gauche, le royaume de la vertu et, à droite, celui du vice. 
Là aussi, comme dans le cas de Ginevra de' Benci, le parcours à accomplir se situe entre extériorité et intériorité, mais le portrait moral est confié à des images on ne peut plus énigmatiques.

Il est fascinant de souligner quelques coïncidences temporelles et géographiques qui concernent Piero della Francesca et Lorenzo Lotto: Bembo restera longtemps à la cour d'Urbin, de I506 à I5I2, mais il y était déjà resté en I504 et cela correspond à une halte dans un voyage à Rome, c'est-à-dire quand il est en train d'écrire les Asolani. Bernardo de' Rossi, le protagoniste du portrait dans le panneau de Lotto était évêque de Trévise, ville dans le territoire duquel se trouvait Asolo. Or, le panneau recouvrant le tableau portait la date du premier juillet I505, année où les Asolani sont publiés. Et surtout, on a formulé une hypothèse très intéressante à propos du portrait de Ginevra de' Benci, qui aurait été commandé à Leonardo par le père même de Pietro Bembo, Bernardo, quand il était ambassadeur à Florence. C'est une hypothèse absolument fascinante pour notre thèse, peut-être trop fascinante, et je me limite à la citer.

En tout cas, il y a un témoignage qui est très intéressant dans notre optique: il s'agit de la discussion entre Bembo et le lettré vicentin Giovan Giorgio Trissino, contenue dans quelques lettres écrites en I505. Il s'agit d'une discussion assez violente à propos d'une médaille (une médaille ancienne probablement) que Trissino ne veut pas donner à Bembo et que Bembo veut absolument. "Je vous dirai que cette médaille en or qui représente Bérénice [...] ressemble de façon frappante à une dame qui existe réellement et que j'bonore beaucoup; c'est pourquoi, pour bien des raisons, si vous me l'aviez donnée, cela aurait été le cadean le plus apprécié que j'aurais jamais pu recevoir de votre part». ${ }^{17}$ Pour avoir cette médaille, Bembo est prêt à donner quelques-unes des "antiquités" dont il est grand collectionneur. ${ }^{18}$ Ce qui est important pour nous, c'est le modèle figuratif qui est ici superposé aux Asolani (Bérénice est en effet une des protagonistes des dialogues): il s'agit d'une médaille avec un portrait de femme. La médaille a un revers, souvent emblématique ou allégorique, c'est pourquoi on peut la considérer comme un précédent, un modèle de la typologie des portraits que nous avons rappelés, c'est-à-dire les portraits "doubles".

Le texte des Asolani a pour point de départ la célébration du mariage d'une demoiselle au service de la reine. Essayons de replacer cette scène initiale dans la complexe machine optique que le texte construit, comme nous l'avons déjà vu; une machine optique qui correspond, au niveau de la structure littéraire, à une structure picturale, raffinée et complexe, entièrement jouée sur la duplicité. La lettre qui dédie les Asolani à Lucrèce Borgia introduit aussi le texte sous une double optique: Bembo écrit, en effet, qu'il lui semble juste d'envoyer son texte alors que Lucrèce est en train de célébrer les noces de "sa gentille Nicola" 
à la Cour de Ferrare: «et il adviendra que vos demoiselles de Cour, vos courtisans et vous-même lirez ce que j'ai écrit ici, moi qui suis tout à vous, c'est-à-dire ce dont d'autres jeunes gens ont parlé avec d'autres dames lors des divertissements d'autres noces» ( $\not p$. 77-78). Les Asolani serviront ainsi d'intermédiaire et de miroir entre deux situations, éloignées l'une de l'autre dans le temps et dans l'espace, comme Bembo qui est loin désormais de la Cour de Ferrare. La mort a fait son entrée dans le royaume de l'amour: la lettre décrit longuement la douleur de Bembo pour la mort de son frère Carlo et fait allusion aux pertes douloureuses que Lucrèce a également subies. Le tableau des festivités pour le mariage à la Cour de la reine de Chypre se recompose ainsi dans l'espace de l'écriture et ne se représente sur la scène d'une nouvelle fête qu'après avoir franchi le seuil obscur de la mort.

Examinons alors comment se présente l'image de la fête nuptiale dans le texte imprimé des Asolani: c'est une image particulièrement mémorable parce qu'elle occupe une place privilégiée, c'est-à-dire l'incipit.

Asolo adunque, vago et piacevole castello posto ne gli stremi gioghi delle nostre alpi sopra il Trivigiano, è, sì come ogniuno dee sapere, di madonna la Reina di Cipri, con la cui famiglia, la quale è detta Cornelia, molto nella nostra città honorata et illustre, è la mia non solamente d'amistà e di dimestichezza congiunta, ma anchora di parentado. Dove essendo ella questo settembre passato a' suoi diporti andata, avenne che ella quivi maritò una delle sue damigielle, la quale, perciò che bella et costumata et gentile era molto et perciò che da bambina cresciuta se l'havea, assai teneramente era da.llei amata et havuta cara. Per che vi fece l'apparecchio delle nozze ordinare bello et grande, et, invitatovi delle vicine contrade qualunque più honorato huomo v'era con le lor donne, et da Vinegia similmente, in suoni et canti et balli et solennissimi conviti l'un giorno appresso all'altro ne menava festeggiando, con sommo piacer di ciascuno.

(I, II, p. 2 I s: "Asolo, donc, beau et agréable château, situé sur la dernière chaîne de nos Alpes et dominant Trévise, appartient, comme chacun le sait, à la Reine de Chypre; sa famille est appelée Cornelia, illustre famille très honorée dans notre ville, unie à la mienne par des liens d'amitié mais aussi de parenté. ${ }^{19}$ En septembre dernier, étant allée là-bas pour se distraire, elle maria une de ses demoiselles de Cour. Celle-ci était très belle, très polie et très aimable et avait grandi à la Cour, c'est pourquoi la reine était très attachée à elle et l'aimait tendrement. Elle ordonna une belle et importante fête de mariage et invita tous les hommes les plus honorables des régions voisines et de Venise ainsi que leurs épouses; ce ne furent que banquets magnifiques, musique, chants, danses, jour après jour, pour le plus grand plaisir de tous").

C'est l'image typique d'une fête de Cour: en un certain sens, la célébration du mariage exalte tout ce qu'ily a de plus raffiné, et à la fois de plus agréable et 
de plus honorable dans la vie de Cour. Tout y est beau, agréable et grand, naturellement. Il n'existe pas d'endroit, ni d'occasion plus convenable pour parler d'amour.

À présent, essayons de soumettre cette image à différents points de vue, en la plaçant à l'intérieur des multiples perspectives que l'on peut construire si l'on fait interagir le passé et le futur, c'est-à-dire si l'on tient compte aussi bien de la version précédente de l'incipit des Asolani que des pages que Bembo, dans sa maturité, allait consacrer à la reine de Chypre.

Dans le texte manuscrit des Asolani, la partie concernant la reine de Chypre est bien plus étendue.

Asolo è hora (sì come dovete sapere) arnese della Reina di Cipri, la quale per la morte del re Giacco suo marito, non guari con lui dimorata, rimasa vedova et sanza figliuoli, poi che così hebbe con somma sodisfattione de' suoi popoli quasi tutta la sua giovanezza trappassata, raccommandato il governo del regno alla Signoria nostra, in Vinegia se ne tornò, dove era nata, per rivedere e suoi parenti et appo loro nella sua patria più riposatamente menarne gli altri anni della sua vita. Dove venuta et lietamente ricevuta da' suoi, fu dalla Signoria nostra honorata di bellissimi et preciosi doni, tra' quali hebbe questo castello, del quale io dico.

(I, II, p. 7: "Asolo (comme vous devez le savoir) appartient à présent à la Reine de Chypre; elle ne vécut pas longtemps avec son mari, le roi Giacco, mais restée veuve et sans enfants, elle passa là-bas presque toute sa jeunesse à la plus grande satisfaction de son peuple. Après avoir confié le gouvernement du royaume à notre Seigneurie, elle revint à Venise, là où elle était née, pour revoir sa famille et passer plus tranquillement avec elle le reste de sa vie. Elle fut bien accueillie par sa famille, notre Seigneurie lui fit honneur en lui offrant des cadeaux précieux et très beaux: parmi ceux-ci, elle reçut le château dont je parle").

Là aussi, le ton est idyllique et le récit, très idéalisé. Toutefois, nous apprenons que la reine qui célèbre le mariage de sa demoiselle de Cour a subi une double perte: celle de son mari et celle de son royaume. Le décor reste splendide et raffiné, mais l'image de la Cour nous apparaît tout à coup fragile et trompeuse: la Cour de Caterina est une Cour que la République de Venise lui a donnée, sur ses propres territoires; il s'agit donc d'une Cour et d'un royaume de carton-pâte, qui remplacent et qui représentent une vraie Cour et un vrai royaume, inexorablement perdus. Lieu idéal de la politesse, de l'élégance du langage et des bonnes manières, dans les Asolani, la Cour devient ainsi, encore plus clairement, un lieu littéraire, le lieu délégué pour parler d'amour, un lieu chargé de ce rapport trompeur et fuyant avec le réel que la littérature sous-entend et qui est un des grands thèmes de l'ouvrage.

Dans la version imprimée des Asolani, l'histoire de la reine de Chypre disparaît: Bembo introduit la référence selon laquelle les Corner provien- 
draient d'une branche de la gens Cornelia ${ }^{20}$ et il souligne à la fois les liens de parenté et d'amitie que sa propre famille aurait avec celle de la reine. La formule «come ogniuno dèe sapere» ("comme chacun doit le savoir") renvoie à une mémoire vénitienne commune; c'est à cette mémoire et non plus au récit qu'est confié le rappel des événements qui précèdent, de ces vicissitudes qui, une fois qu'elles sont présentées directement sur la scène, risquent de créer une atmosphère de précarité autour de la Cour et des rites qui y sont célébrés.

En I530, le Conseil des Dix charge Bembo d'écrire l'histoire de Venise: l'ouvrage, en latin, sera achevé en I544, quand Bembo est devenu cardinal. Nous y trouvons une version bien plus longue et plus mouvementée des vicissitudes de la reine de Chypre. Un véritable roman, fait d'intrigues internationales, d'espionnage et de trabisons se dessine autour de la figure féminine qui avait célébré, au début des Asolani, les noces d'une de ses demoiselles de Cour, dans une atmosphère paradisiaque. Arrêtons-nous sur les éléments qui nous intéressent, parce qu'ils renvoient à la scène construite dans les Asolani. Nous apprenons que le roi de Naples aurait voulu marier son fils avec Caterina Corner et que la République de Venise empêche par la force ce mariage. Contrairement à ce qui est raconté dans la première version des Asolani, Caterina ne voulait pas abandonner le royaume de Chypre; nous pouvons lire: «et elle ne voulait pas qu'on la persuade de quitter un riche royaume, car c'était une femme babituée aux honneurs et à une vie de reine et elle savait comment sans reconnaissance de son rang on vivait sobrement dans les républiques». Son frère Giorgio, le chef de la délégation vénitienne, la convainc d'accepter grâce à de nombreux arguments, farcis de flatteries et de menaces parmi lesquels figure aussi la remarque que les notables de l'ille ne supportent pas d'être gouvernés par une femme: «et de nombreux notables de Chypre sont ouvertement indignés d'être gouvernés par une femme». L'exhortation que son frère adresse à Caterina est très intéressante; il l'invite à renoncer à l'essence même de son image publique, pour n'en montrer que le nom et la splendeur extérieure dans sa patrie d'origine: "Vous serez. nommée reine dans toute région [...] vous devriez aussi désirer être saluée et vue comme reine dans votre patrie; vous devriez vouloir montrer votre gloire à votre frère, à vos scurs, à vos parents, à votre famille et à la ville où vous êtes née et où vous aver grandi》. ${ }^{21}$

C'est ce que représentera justement Asolo: le triomphe de la pure image, c'est-à-dire la représentation d'une gloire à laquelle ne correspond que le souvenir d'une réalité.

Du reste, pour toute personne en possession d'une culture moyenne, évoquer Chypre et sa reine signifiait évoquer Vénus et son royaume. La reine Corner qui célèbre les noces d'une de ses demoiselles de Cour et qui, par sa présence, donne une conclusion et du prestige à ce «ragionar d'amore» est donc aussi 
l'image emblématique de la déesse de l'amour. Le rapport entre les deux portraits, l'un réaliste, l'autre emblématique, peut être lu de différentes façons: nous pouvons le reconduire à l'éloge courtisan et au code mythologique qui lui est cher ('cest ce qui arrive par exemple dans le Cabinet d'Isabelle d'Este qui est réalisé à l'époque où Bembo écrit les Asolani; le tableau que Lorenzo Costa livre, en I506, représente Isabelle couronnée au royaume d'Amour), ${ }^{22}$ ou bien nous pouvons lire le rapport entre la reine de Chypre et la reine de l'Amour comme un expédient qui recouvre un vide, qui cache un manque. D'ailleurs, comme le rappelle Gismondo, l'alter ego de l'auteur, il existe un plaisir de la mémoire qui se nourrit justement de l'absence, de la privation, et qui unit l'amoureux et le poète:

O Amore, benedette sieno le tue mani sempre da me, con le quali tante cose m'hai dipinte nell'anima, tante scritte, tante segnate della mia dolce donna, che io una lunga tela porto meco ad ogni hora d'infiniti suoi ritratti in vece d'un solo viso, et un alto libro leggo sempre et rileggo pieno delle sue parole.

(II, xxvII, pp. 298-99: “Oh Amour, que je bénisse toujours tes mains, grâce auxquelles, dans mon esprit, tu as peint, tu as écrit, tu as dessiné tellement de choses inspirées par ma douce dame que j'ai toujours avec moi une longue toile avec ses portraits infinis et non pas un seul, et que je lis et je relis toujours un ouvrage épais rempli de ses mots").

Bembo décline ici le topos ancien de l'image de la femme aimée qui est peinte dans le coeur: le fait qu'il le décline au pluriel en multipliant à l'infini les portraits et les mots d'amour qui y sont liés correspond bien à la structure des Asolani que nous avons essayé de reconstruire.

Pour conclure, j'ai pensé que souligner l'analogie entre la structure des Asolani et celle des doubles portraits peut aider à comprendre combien l'image de la cour, de la littérature, de l'amour que le texte de Bembo construit est difficile à interpréter: il s'agit d'une image qui ne peut pas se réduire à une seule perspective, mais qui demande aux observateurs d'adopter des points de vue différents. De toute façon, nous pouvons rappeler l'observation de Montaigne: "Un suffisant lecteur descouvre souvant ès escrits d'autruy des perfections autres que celles que l'autheury a mises et appercues, et y preste des sens et des visages plus riches». ${ }^{23}$ Nous ne nous considérons certainement pas comme des «lecteurs suffisants», mais nous avons voulu accepter l'invitation de Montaigne à nous faire prendre par l'enchantement du texte et à collaborer avec lui.

Lina Bolzoni 


\section{Lina Bolzoni}

Cet article est le texte de la conférence que j'ai tenue le I 2 mai 2005 à l'Université de Genève, pour la Fondation Barbier-Mueller. Je désire remercier très sincèrement le Conseil de la Fondation de m'avoir invitée, me permettant ainsi de vivre une expérience inoubliable.

I. Nous disposons d'une édition critique des Asolani, Pietro Bembo, Gli Asolani, sous la dir. de G. Dilemmi, Firenze, Accademia della Crusca I99I, d'où sont extraites toutes mes citations. Je signale aussi que, dans la bibliothèque du Wellesley College (Mass.), est conservé un exemplaire des Asolani de i 505 avec des corrections autographes (Catalogue of the Frances Taylor Pearsons Plimpton Collection, Cambridge Mass., Harvard University Press, I929, p. 46). Il existe une excellente monographie, celle de C. Berra, La scrittura degli "Asolani" di Pietro Bembo, Firenze, La Nuova Italia, 1995, que l’on peut compléter avec l'excellente analyse rhétorique et littéraire de F. Finotti, Retorica della diffrazione. Bembo, Aretino, Giulio Romano e Tasso: letteratura e scena cortigiana, Firenze, Olschki, 2004, chap. I et chap. II. À propos des Asolani voir en outre l'introduction de C. Dionisotti à Pietro Bembo, Prose e rime, Torino, UTET, r 960, pp. 9-56; l'introduction de M. Marti à Pietro Bembo, Opere in volgare, Firenze, Sansoni, 196I, pp. XI-XVIII; L. Baldacci, Gli "Asolani" del Bembo e Venere celeste, dans Il petrarchismo italiano nel Cinquecento, Padova, Liviana, I 974 (II edizione), pp. 8 5- I Iо; P. Floriani, Bembo e Castiglione. Studi sul classicismo del Cinquecento, Roma, Bulzoni, Roma I976, pp. 78-98; N. Ordine, Il dialogo cinquecentesco italiano tra diegesi e mimesi, dans «Studi e problemi di critica testuale», XXXVII (1988), pp. I55-79 (cf. p. I62 sq.); F. Tateo, La disputa dell' amore: retorica e poetica del contrario, dans Il dialogo filosofico nel Cinquecento europeo, sous la dir. de D. Bigalli et G. Canziani Milano, Angeli, I990, pp. 209-28; P. Sabbatino, Gli "Asolani". La letteratura sulla scena del giardino di corte, dans La "scienza" della scrittura. Dal progetto del Bembo al manuale, Firenze, Olschki, 1988, pp. 13-45; R. Scrivano, La forma del dialogo: Pietro Bembo, dans Il modello e l'esecuzione, Roma, Bulzoni, pp. Iо3-I 2; M. Pozzi, Aspetti della trattatistica d'amore, et Pietro Bembo, dans Lingua, cultura, società. Saggi della letteratura italiana del Cinquecento, Alessandria, Edizioni dell'Orso, 1989, p. 57 sq. et pp. 170-204; C. Forno, Il "libro animato": teoria e scrittura del dialogo nel Cinquecento, Torino, Tirrenia Stampatori, 1992; T. Cachey, "Il pane del grano e la saggina": Pietro Bembo's isos "Asolani" revisited, dans "The Italianist», XIII (1993), pp. 5-23; L. Bolzoni, La stanza della memoria, Modelli letterari e iconografici nell' età della stampa, Torino, Einaudi, I995, pp. 88-89 et pp. I 87-89; L. Bolzoni, Memoria e gioco nella letteratura del Cinquecento: gli "Asolani" e altri esempi, in Studi offerti a Luigi Blasucci dai collegbi e dagli allievi pisani, Lucca, Pacini Fazzi, I 996, pp. I I 5-32; L. Bolzoni, Il giardino e il bosco, ovvero il "ritratto doppio" della campagna negli "Asolani", in La campagna in città. Letteratura e ideologia nel Rinascimento. Studi in onore di Michel Plaisance, sous la dir. de Giuditta Isotti Rosowsky, Firenze, Franco Cesati editore, 2002, pp. 8 I-I 04 . On peut aussi trouver quelques allusions dans A. Godard Le dialogue à la Renaissance, Paris, PUF, $200 \mathrm{I}$ et V. Cox, The Renaissance Dialogue: Literary dialogue in Its Social and Political Context, Cambridge, Cambridge University Press, 1992.

2. Pour cette lecture, cf. la notice de C. Dionisotti, Pietro Bembo, in Dižionario biografico degli italiani, vol. VIII, Roma, Istituto dell'Enciclopedia italiana, I 966, pp. I33-50. Cf. en outre la reconstruction de la figure du père de Pietro Bembo dans N. Giannetto, Bernardo Bembo umanista e politico veneziano, Firenze, Olschki, 1985.

3. Cf. Maria Savorgnan - Pietro Bembo, Carteggio d'amore (isoo-isoi), sous la dir. de C. Dionisotti, Firenze, Le Monnier, 1950. À propos de l'édition des lettres de M. Savorgnan, cf. A. E. Quaglio, Intorno a Maria Savorgnan: per una riedizione delle lettere, dans "Quaderni utinensi», V-VI (1985), pp. 103-I8 et Intorno a Maria Savorgnan. Un "sidio" d'amore, dans «Quaderni utinensi», VII-VIII (1986), pp. 77-Iог. Cf. en outre G. Dilemmi, "Andrem di pari": Maria Savorgnan e "Gli Asolani" del Bembo, in «Quaderni del Dipartimento di Lingue e Letterature neolatine», Università di Bergamo, IV (1988-1989), pp. 49-72. Il est possible de lire les lettres de Bembo dans l'ouvrage: PIETro BemBo, Lettere (I492-I507), I, édition critique sous la dir. de E. Travi, Bologna, Commissione per i testi di Lingua, 1987. 
4. Cf. Pietro Bembo - Lucrezia Borgia, La grande fiamma. Lettere I503-I5I7, sous la dir. de G. Raboni, Milano, Archinto, 1989 .

5. Cf. C. H. Clough, P. Bembo's "Gli Asolani” of I505, dans «Modern Languages Notes», LXXXIV (1969), pp. I6-45 et LXXXVII (1972), pp. I 34-38 et C. Fahy, $A$ note of the printing of the Isos Aldine edition of P. Bembo's "Asolani", dans «The Library», XXXVIII (1972), pp. I36-42.

6. Cf. les observations de M. L. Doglio, Introduzৃione à G. F. CAPRA, Della eccellenz̧a e dignità delle donne, Roma, Bulzoni, 1988, pp. 5-53 (cf. pp. 8-10).

7. G. Arbizzoni, L'ordine e la persuasione. Pietro Bembo personaggio nel "Cortegiano", Urbino, Quattro Venti, ${ }_{9} 983$; T. Cachey, In and out of the margins of a Renaissance Controversy: Castiglione in the second "Asolani" (I530), dans «Rivista di letteratura italiana», I (1985), pp. $253-62$.

8. Je tiens à préciser ici qu'au cours de la discusion qui a suivi ma conférence, Guglielmo Gorni a livré une suggestion pertinente à propos de la lecture de ce vers: dans «utile scampo a le mie pene imPETRO», il décèle l'indice qui renvoie à l'histoire d'amour du poète.

9. Cf. G. Gorni, Ragioni metriche della canzone, tra filologia e storia, dans Studi di filologia e di letteratura italiana offerti a C. Dionisotti, Milano-Napoli, Ricciardi, 1973 et S. Carrai, I precetti di Parnaso. Metrica e generi poetici nel Rinascimento italiano, Roma, Bulzoni, I999.

Io. À propos du jardin et de sa fonction assumée dans le dialogue, cf. G. Venturi, "Picta poesis": ricerche sulla poesia e il giardino dalle origini al Seicento, dans Storia d'Italia, Annali 5, Il paesaggio, sous la dir. de C. De Seta, Torino, Einaudi, I982, pp. 669-749 (cf. pp. 698-703); Sabbatino, Gli "Asolani" cit., p. 33; Berra, La scrittura cit., pp. 2 I 4- I6. Au sujet des jardins de la demeure des Bembo et de Caterina Corner, cf. L. Puppi, Le residenze di P. Bembo in "padoana", dans «L'arte», II (I 979), 7-8, pp. 30-66; M. Fagiolo, Il giardino come teatro del mondo e della memoria, in La città effimera e l'universo artificiale del giardino, sous la dir. de M. Fagiolo, Roma, Officina, I980, pp. г 25-41; L. Puppi, Il "barro" di Caterina Cornaro ad Altivale, dans «Prospettive», 25 (1962), pp. 52-64.

I I. Dans la version manuscrite, le texte des deux chansons est différent. Les capacités mnémotechniques que la structure choisie impose à Gismondo est commentée de cette façon, avec une sorte de mise en abyme ironique: «Maravigliavansi le donne, recitate queste canzoni, come Gismondo così bene si ricordasse que' versi che una volta sola uditi cantare havea» (I, v, pp. I4-I 5: "Après avoir écouté ces chansons, les dames s'étonnaient que Gismondo se souvienne aussi bien de ces vers, des vers qu'il n'avait entendus qu'une seule fois").

I 2. Au sujet des rapports entre les Asolani et la peinture de l'époque, dans une optique différente de celle qui est proposée ici, cf. G. Romano, Verso la maniera moderna: da Mantegna a Raffaello, dans Storia dell'arte, II, 2, Torino, Einaudi, 1981, pp. $5-88$ (cf. p. 23 sq.) et A. Ballarin, Giorgione e la Compagnia degli Amici: il "Doppio ritratto" Ludovisi, in Storia dell'arte italiana, sous la dir. de F. Zeri, I, Dal Medioevo al Quattrocento, Torino, Einaudi, I983, pp. 479-54I.

13. Un instrument de travail très utile est le catalogue de Angelica Dulberg, Porträts: Geschicbte und Ikonologie einer Gattung in I5. und 16. Jabrbundert, Berlin, Mann, 1990. Je ne connais aucune étude qui reconstruise le "genre" dans ses formes variées, elle serait pourtant d'un grand intérêt. L'ouvrage de référence est toujours celui de J. Pope-Hennessy, The Portrait in the Renaissance, New York, Phaidon Press, 1966, notamment le chap. IV, où il montre comment le goût pour les portraits des empereurs figurant sur les monnaies 


\section{LinA Bolzoni}

romaines influence le portrait de profil et surtout le chap. V, Image and emblem, où il souligne l'importance du modèle de la médaille (côté pile et côté face), et le lien étroit entre le 'portrait double' et la typologie du portrait avec un emblème. On peut citer aussi l'étude de L. Campbell, Frames and covers, in Renaissance Portraits. European Portrait-Painting in the $14^{t h}, I 5^{t h}$ and $I^{\text {th }}$ Centuries, New Haven-London, Yale University Press, 1990, pp. 65-67. Cf. en outre The Image of the Individual: Portraits in the Renaissance, sous la dir. de Nicolas Mann et Luke Syson, London, British Museum Press, 1998; Jodi Cranston, The Poetics of Portraiture in the Italian Renaissance, Cambridge, Cambridge University Press, 2000; John Roger Paas, Effigies et poesis: an Illustrated Catalogue of Printed Portraits with Laudatory Verses by German Baroque Poets, Wiesbaden, Harrasowitz, I 998; American Library Association, ALA Portrait Index: Index to Portrait Contained in Printed books and Periodicals, Washington, Library of Congress, 1906; Heidi Ebertshauser, Gattungen des Doppelportrats in der Deutschen Malerei des 16. Jabrbunderts, s.l.sn., I 974 .

14. Cf. pour d'autres références bibliographiques, dans Piero e Urbino, Piero e le Corti rinascimentali, sous la dir. de P. Del Poggetto, Venezia, Marsilio, ı992: C. Cieri Via, I trionfi di Piero, pp. I 26-34 e M. C. Castelli, Piero della Francesca. Ritratti e trionfi allegorici di Federico da Montefeltro e Battista Sforza (Dittico), pp. I 54-58; J. Woods Marsden, Piero della Francesca's Ruler Portraits, in The Cambridge Companion to Piero della Francesca, ed. by J. M. Wood, Cambridge, Cambridge University Press, 2002, pp. 9I-I I 4.

I 5. Cf. P. C. Marani, Leonardo. Una carriera di pittore, Milano, Federico Motta, I999, pp. 38-48 et Virtue and Beauty, sous la dir. de D. A. Brown, Washington 200 r pour d'autres références bibliographiques.

I6. Le portrait de l'évêque est conservé aujourd'hui au Musée de Capodimonte, à Naples, tandis que le "couvercle" se trouve à la National Gallery de Washington. Cf., pour d'autres références bibliographiques, D. A. Brown, Ritratto del vescovo Bernardo de' Rossi et Allegoria della Virtù e del Vižio, in Lorenzo Lotto. Il genio inquieto del Rinascimento, Milano, Skira, I998, pp. 73-80 et A. Gentili (avec M. Lattanzi et F. Polignano), I giardini di contemplazione: Lorenzo Lotto I503-I5I2, Roma, Bulzoni, 1985.

17. BEMBO, Lettere (I492-I507), lettre n. 202, du 2I mars i 505 , p. 190.

I 8. Au sujet de la passion numismatique de Bembo, cf. D. Gasparotto, La barba di P. Bembo, dans «Quaderni degli Annali della Scuola Normale Superiore», Studi in onore del Kunsthistorisches Institut in Florenz per il suo centenario (1897-1997), pp. 183-206. Pour un tableau de la numismatique au XVI ${ }^{\mathrm{e}}$ siècle, cf. J. Cunnally, Images of the Illustrious. The Numismatic Presence in the Renaissance, Princeton, Princeton University Press, I999 et le répertoire de George Francis Hill, A Corpus of Italian Medals of the Renaissance before Cellini, (London, I930), Firenze, Studio per Edizioni Scelte, I984; Italian Medals, sous la dir. de J. Graham Pollard, Washington-Hanover (N.H.), National Gallery of Art-University Press of New England, 1987; The Currency of Fame: Portrait/Medals of the Renaissance, London, Thames and Hudson 1994 .

19. À propos de Caterina Corner, cf. F. Colasanti, Caterina Corner (Cornaro), in Dizionario biografico degli italiani, vol. 22, Roma, Istituto dell'Enciclopedia italiana, 1979, pp. 33542.

20. Le lien avec la gens Cornelia correspondait à une étymologie courante qui était employée pour anoblir la famille Corner. Un autre témoignage est constitué par l'inscription qui accompagne le portrait de Caterina Corner peint par Gentile Bellini et qui se trouve actuellement au Musée de Budapest (cf. J. Pope-Hennessy, The Portrait cit., p. 50 et note 7I, p. 309 avec le texte de l'inscription). 
Les Asolani de Pietro Bembo

2. Pietro Bembo, Della istoria viniziana [...] da lui volgarizzata libri dodici, in Opere, vol. III, Milano, Società Tipografica dei Classici italiani, I809, pp. 63-65.

22. C'est Romano qui le rappelle, Verso la maniera cit., p. 38, le considérant très proche des Asolani.

23. Michel de Montaigne, Les Essais, sous la dir. de P. Villey, Paris, Presses Universitaires de France, 1978 , livre I, 24, p. I 27. 